\title{
Echocardiographic evaluation including tissue Doppler imaging in New Zealand white rabbits sedated with ketamine and midazolam is
}

\author{
Ana Patrícia Fontes-Sousa ${ }^{\mathrm{a}, \mathrm{b}}$, Cláudia Moura ${ }^{\mathrm{a}}$, Catarina Santos Carneiro a \\ Armando Teixeira-Pinto ${ }^{c}$, José Carlos Areias ${ }^{a}$, Adelino F. Leite-Moreira ${ }^{\mathrm{a}, *}$ \\ a Department of Physiology, Faculty of Medicine, University of Porto, 4200-319 Porto, Portugal \\ ${ }^{\mathrm{b}}$ Department of Veterinary Sciences, University of Trás-os-Montes e Alto Douro, 5001-801 Vila Real, Portugal \\ ${ }^{\mathrm{c}}$ Department of Biostatistics and Medical Informatics, CINTESIS, Faculty of Medicine, University of Porto, 4200-319 Porto, Portugal
}

Accepted 26 February 2008

\begin{abstract}
Limited data are available on the use of more recent echocardiographic parameters in the rabbit. Echocardiographic examination, including conventional echocardiography and tissue Doppler imaging (TDI), was performed on 26 male New Zealand white rabbits under ketamine-midazolam sedation. Particular emphasis was placed on the more recent systolic and diastolic parameters, such as myocardial performance index (Tei index) and mitral annular motion (from septal and lateral sides of the left ventricle) obtained using pulsed TDI.

Parameters that assessed systolic and diastolic function (fractional shortening, Tei index, and maximal mitral $E$ - and $A$-wave velocities) were comparable to those reported in the literature for rabbits in the awake state. The less cardiodepressive anaesthetic protocol could offer a good alternative in performing echocardiographic evaluation whenever such caution is necessary. TDI is feasible in healthy rabbits and potentially suitable for the investigation of left ventricle systolic and diastolic function.
\end{abstract}

(c) 2008 Elsevier Ltd. All rights reserved.

Keywords: Doppler echocardiography; Tissue Doppler Imaging; Reference value; Rabbit; Anaesthesia

\section{Introduction}

Cardiac disease has been described in pet rabbits (Martin et al., 1987) and the species is widely used in cardiovascular research (Bras-Silva et al., 2006; Lange et al., 2006; Barraud et al., 2007). Echocardiography is a useful noninvasive method for the in vivo evaluation of ventricular dimensions and performance in experimental and clinical settings.

Doppler echocardiography provides useful additional information on cardiac conditions in both humans and small animals. Parameters obtained with tissue Doppler

\footnotetext{
Presented in part at the 17th European College of Veterinary Internal Medicine Companion Animal Congress, Budapest, September, 2007.

* Corresponding author. Tel.: +35122 5513644; fax: +351 225513646.

E-mail address: amoreira@med.up.pt (A.F. Leite-Moreira).
}

imaging (TDI) have been shown to be more independent of pre- and after-load than classic haemodynamic Doppler measurements and can be used to quantify regional myocardial function accurately and more objectively (Sohn et al., 1997; Firstenberg et al., 2001; Nagueh et al., 2001). Pulsed TDI of the mitral annulus and myocardial wall has been suggested as a means to assess systolic and diastolic left ventricular (LV) function in human and veterinary medicine (Oki et al., 1999; Chetboul et al., 2005; Teshima et al., 2005; Chetboul et al., 2006; O'Sullivan et al., 2007). The Tei index, a new parameter to assess myocardial performance, has been proposed for the assessment of global cardiac performance (systolic and diastolic function) in a wide variety of congenital and acquired cardiac abnormalities (Dujardin et al., 1998; Bruch et al., 2002; Haque et al., 2002; Harjai et al., 2002; Gaibazzi et al., 2005; Dyer et al., 2006). 
Reference values for various M-mode, flow Doppler and tissue Doppler echocardiographic parameters have been reported in rabbits in the conscious state as well as during different anaesthetic combinations (Fontes-Sousa et al., 2006; Stypmann et al., 2007). Nevertheless, examination of the awake rabbit is more difficult, more time-consuming, and needs special training, especially with research animals or animals less accustomed to handling. Anaesthesia can be used but might affect cardiac function, and the extent will depend on the type of anaesthesia (Schaefer et al., 2005). It is therefore important to know the effect of standardised sedation protocols on echocardiographic parameters. Recent studies have used ketamine- $\alpha_{2}$ agonist combinations to perform echocardiography in rabbits (FontesSousa et al., 2006; Stypmann et al., 2007) but the major obstacle with this combination is its potential for cardiac and respiratory depression (Sanford and Colby, 1980). As an alternative, ketamine in combination with midazolam, a short-acting benzodiazepine, has been described for chemical restraint in rabbits associated with minimal cardiorespiratory depression (Dupras et al., 2001).

The purpose of the present study was to determine reference values for echocardiographic M-mode, Doppler, and pulsed TDI measurements in clinically healthy New Zealand white rabbits sedated with ketamine and midazolam.

\section{Materials and methods}

The study was performed according to the Portuguese Law for Animal Welfare. The investigation conformed to the guide for the care and use of laboratory animals published by the US National Institutes of Health (NIH Publication No. 85-23, revised 1996).

Twenty-six young adult healthy male New Zealand white rabbits (1620 weeks old and weighing $2.3 \pm 0.4 \mathrm{~kg}$ ) were used. Rabbits were healthy and free from any sign of cardiovascular or respiratory tract disease on the basis of a physical examination that included careful thoracic auscultation and normal echocardiogram. The animals were housed in stainless steel cages in a controlled environment, at temperatures of $20-25^{\circ} \mathrm{C}$, with a 12:12 h light dark cycle, and were fed with a standard pellet diet and water ad libitum. The weight of each rabbit was recorded prior to anaesthesia.
A combination of ketamine-hydrochloride $(20 \mathrm{mg} / \mathrm{kg}$; Imalgene 1000 , Merial) and midazolam ( $2 \mathrm{mg} / \mathrm{kg}$; Midazolam APS, Farma-APS) was administered SC to each rabbit to minimise defensive movements and facilitate complete echocardiographic examination. Typically, the rabbits were completely immobilised within $5-10 \mathrm{~min}$.

Echocardiography was carried out under light anaesthesia and spontaneous respiration, using a GE Vivid 7 system (GE VingMed) equipped with tissue Doppler technology. The standard phased-array, variablefrequency $(3.5-6.9 \mathrm{MHz})$ transducer was used for two-dimensional, Doppler, and TDI recordings. Recordings were made under continuous ECG monitoring (lead II) by fixing the electrodes on the limbs at a sweep speed of 100 and $200 \mathrm{~mm} / \mathrm{s}$ for off-line analysis. All echocardiographic acquisitions were made in sinus rhythm.

Rabbits were placed in right or left lateral recumbency to obtain right and left parasternal views, respectively, over a gap in the tabletop through which the ultrasound probe was brought from below and placed on a shaved area on the anterior aspect of the lower portion of the thoracic wall. Echocardiographic measurements were obtained from standard views (Thomas et al., 1993).

From the right parasternal short-axis view, two-dimensional guided M-mode tracings were made just below the mitral valve at the level of the papillary muscles for measurements of the interventricular septum (IVS), left ventricular internal diameter (LVID), and left ventricular free wall (LVFW) in diastole and systole. The right parasternal long-axis view with two-dimensional guided M-mode was used for the measurements of the Epoint-to-septal separation interval in the plane of mitral valves. In this same view, the aortic and left atrial diameters were evaluated at the level of the aortic valve. These measurements were obtained applying the leadingedge method of the American Society of Echocardiography (Sahn et al., 1978). Left ventricular ejection fraction was calculated by use of the cube method according to the formula: ejection fraction $=\left[\left(\mathrm{LVIDd}^{3}-\right.\right.$ $\left.\left.\mathrm{LVIDs}^{3}\right) / \mathrm{LVIDd}^{3}\right] \times 100$.

Doppler examinations were performed according to protocols established for dogs and cats (Gaber, 1991). Heart rate was calculated directly from the pulsed Doppler tracings. Variables recorded for each rabbit included maximal pulmonary artery and aortic outflow velocity, namely the maximal pulmonary outflow velocity $\left(\mathrm{PA}_{\max }\right)$ and maximal aortic outflow velocity $\left(\mathrm{Ao}_{\max }\right.$ ), maximal $E$ - and $A$-wave velocities, $E$ : $A$ ratio, isovolumetric relaxation time (IVRT), isovolumetric contraction time (IVCT), left ventricle ejection time (LVET) and Tei index.

The velocities were recorded as the maximal value on the outer edge of the peak velocity spectrum. Pulmonary artery flow velocity was determined by use of pulsed Doppler from the right parasternal short-axis view. The velocities of aortic flow and mitral flow-peak early diastolic wave $(E)$ and peak atrial contraction wave $(A)$ were recorded via pulsed Doppler from the
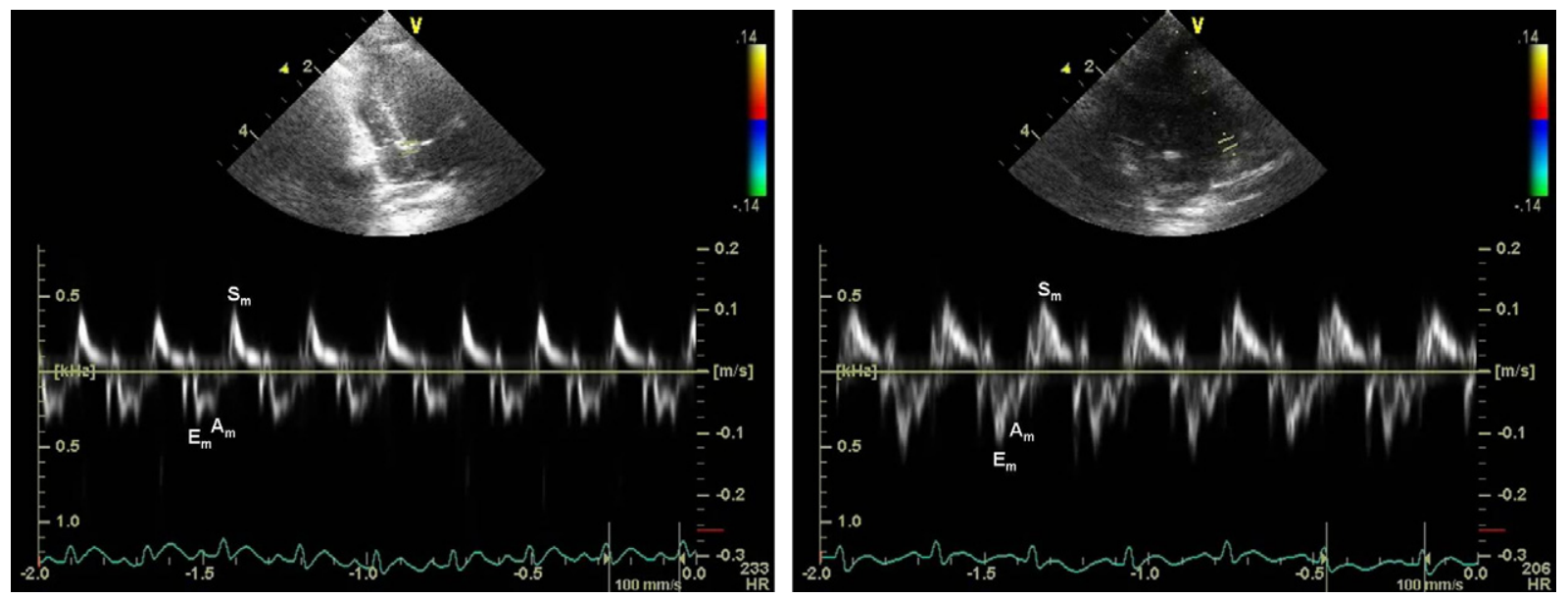

Fig. 1. The velocity profiles ( $\left.E_{\mathrm{m}}, A_{\mathrm{m}}, S_{\mathrm{m}}\right)$ obtained from pulsed tissue Doppler imaging of septal (left) and lateral (right) mitral annulus in rabbits. The values of $E_{\mathrm{m}}$ peak velocities are significantly different between septal (lower values) and lateral side (higher values) of the mitral annulus. $E_{\mathrm{m}}$ : the peak early diastolic velocity. $A_{\mathrm{m}}$ : the peak atrial diastolic velocity. $S_{\mathrm{m}}$ : the peak systolic velocity. 
left parasternal apical 5- and 4-chamber views. Mitral inflow velocity pattern was recorded with the sample volume between the tips of the leaflets. In the great vessels, the sample volume was positioned in the centre of the vessel, just beyond the valve leaflets, and colour Doppler was used to help align the cursor parallel to blood flow. Alignment was maximised in the two-dimensional view and no angle of correction was used.

IVRT was measured as the time interval between end of aortic outflow and onset of the mitral inflow by pulsed Doppler. IVCT was measured as the time interval between the end of mitral inflow and onset of aortic outflow by pulsed Doppler. The Tei index was calculated as described, Tei $=($ IVCT + IVRT $) /$ LVET $($ Tei et al., 1995).

TDI was performed from the left parasternal apical 4-chamber view as previously described (Nagueh et al., 2001; Gan et al., 2004). In brief, the mitral annular motion was measured from the septal and lateral (free wall) side with pulsed TDI. Colour TDI was used to aid in sample volume placement, and the cursor was aligned as parallel as possible to the longitudinal axis of LV wall motion. Gain and filter settings were adjusted to eliminate background noise and to allow the recording of clear tissue signals. Measurements included peak early diastolic $\left(E_{\mathrm{m}}\right)$, late diastolic $\left(A_{\mathrm{m}}\right)$ and systolic $\left(S_{\mathrm{m}}\right)$ mitral annular velocities (Fig. 1), with calculation of $E_{\mathrm{m}}: A_{\mathrm{m}}$ and $E: E_{\mathrm{m}}$ ratios.

All data were collected by use of a trackball-driven cursor and ultrasound system software. The measured beats were selected on the basis of quality of the recording and presence of a regular cardiac rhythm. For each parameter the mean of three representative cardiac cycles was recorded. From these means, the overall mean, standard deviation (SD), and range for all variables measured in all rabbits were calculated. All images were stored digitally on optical discs and analysed retrospectively. The measurements were performed offline using dedicate software (EchoPAC 7).

\section{Statistical analysis}

The statistical analysis was performed using the software SPSS for Windows, 15.0. Mean values, SD, maximum and minimum values (range) and percentiles for the echocardiographic parameters were computed. Pearson correlation coefficients $(r)$ were used to study the association between rabbit bodyweights and heart rates and their respective mean Mmode and Doppler echocardiographic measurements. The $P$-level for statistical significance was set at 0.05 .

\section{Results}

All 32 echocardiographic measurements (M-mode, 2D, Doppler echocardiography and TDI) were easily recorded in all rabbits in order to obtain reference values for the breed when sedated with ketamine-midazolam. No animal died during or after the examination. Mean bodyweight was $2.2 \mathrm{~kg}(\mathrm{SD}=0.4$, range $1.9-3.5 \mathrm{~kg})$. Mean $( \pm \mathrm{SD})$ heart rate was $262 \pm 37 \mathrm{bpm}$ and remained stable throughout the examination. Recording was typically completed approximately 20 min after administration of ketamine and midazolam. $A$-wave was usually superimposed to $E$-wave due to the elevated heart rate, but it was possible to distinguish between the peak velocity of $E$ - and $A$-wave with normal or higher frame rates (Fig. 2).

Tables 1 and 2 summarise the results of the two-dimensional and M-mode measurements, Doppler echocardiography including conventional Doppler and TDI. We found that heart rate correlated with few echocardiographic parameters. A weak significant negative correlation was found between heart rate and LVIDs $(r=0.50, P=$ 0.01 ). Weak positive correlations were found between heart

\section{Table 1}

Values for two-dimensional and M-mode echocardiographic variables in 26 male New Zealand white rabbits sedated with a combination of ketamine and midazolam

\begin{tabular}{|c|c|c|c|}
\hline Parameter & Mean \pm SD & 5 th percentile & 95th percentile \\
\hline $\mathrm{IVS}_{\mathrm{d}}(\mathrm{mm})$ & $2.65 \pm 0.31$ & 2.23 & 3.20 \\
\hline $\operatorname{IVS}_{\mathrm{s}}(\mathrm{mm})$ & $3.63 \pm 0.34$ & 2.97 & 4.13 \\
\hline $\mathrm{LVID}_{\mathrm{d}}(\mathrm{mm})$ & $13.51 \pm 1.05$ & 11.97 & 15.23 \\
\hline $\operatorname{LVID}_{\mathrm{s}}(\mathrm{mm})$ & $8.64 \pm 0.82$ & 7.37 & 10.00 \\
\hline $\mathrm{LVFW}_{\mathrm{d}}(\mathrm{mm})$ & $2.25 \pm 0.29$ & 1.90 & 2.77 \\
\hline $\mathrm{LVFW}_{\mathrm{s}}(\mathrm{mm})$ & $3.15 \pm 0.38$ & 2.60 & 3.93 \\
\hline FS $(\%)$ & $36.01 \pm 4.31$ & 31.18 & 42.83 \\
\hline EF $(\%)$ & $69.58 \pm 5.33$ & 62.99 & 77.73 \\
\hline Ao $(\mathrm{mm})$ & $6.57 \pm 0.46$ & 5.87 & 7.43 \\
\hline $\mathrm{LA}(\mathrm{mm})$ & $7.49 \pm 1.14$ & 5.90 & 9.50 \\
\hline LA:Ao & $1.15 \pm 0.19$ & 0.82 & 1.43 \\
\hline EPSS (mm) & $1.41 \pm 0.25$ & 1.13 & 1.83 \\
\hline
\end{tabular}

$\mathrm{SD}$, standard deviation; $\mathrm{IVS}_{\mathrm{d}}$ and $\mathrm{IVS}_{\mathrm{s}}$, thickness of the interventricular septum in diastole and systole, respectively; LVID $_{d}$ and LVID , left ventricular internal diameter in diastole and systole, respectively; $\mathrm{LVFW}_{\mathrm{d}}$ and $\mathrm{LVFW}_{\mathrm{s}}$, thickness of the left ventricular free wall in diastole and systole; FS, fractional shortening; EF, ejection fraction; Ao, aorta diameter; LA, left atrial diameter; and EPSS, $E$-point to septal separation.
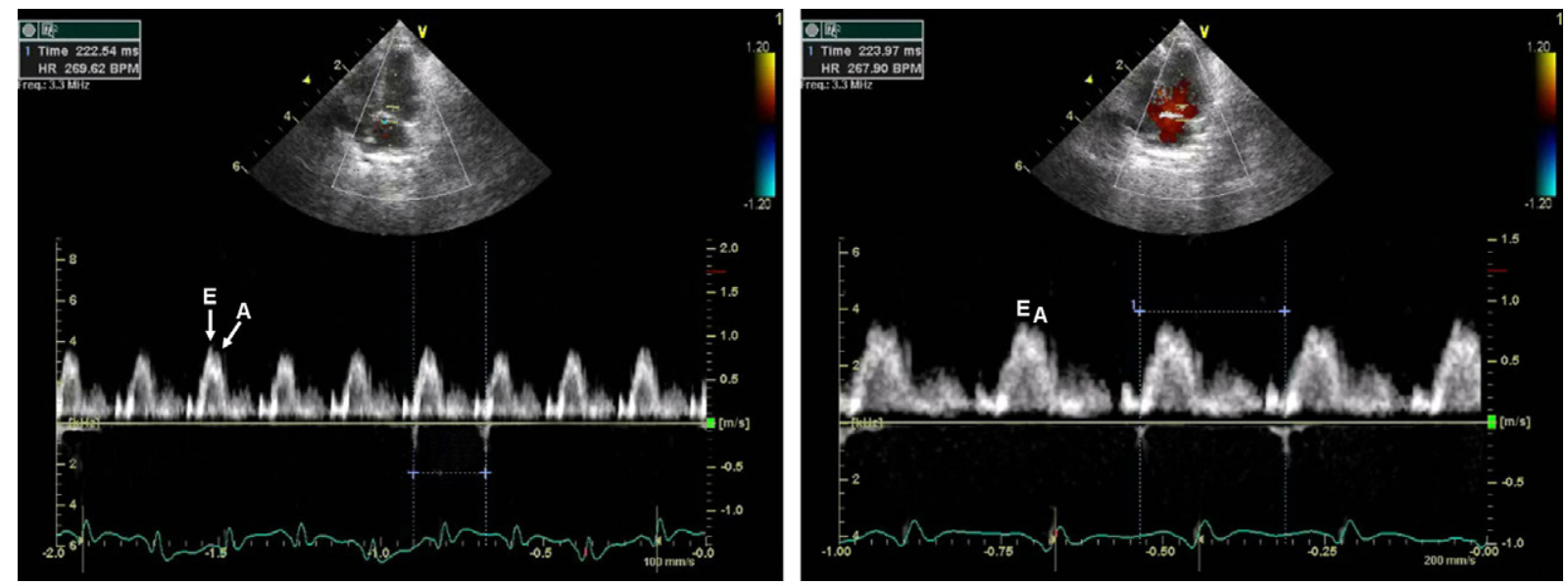

Fig. 2. Pulsed Doppler recordings of left ventricle inflow depicting the peak velocity of $E$ - and $A$-waves, obtained at normal (left) and higher (right) sweep speeds from a rabbit with a heart rate above $250 \mathrm{bpm}$. 
Table 2

Doppler echocardiographic measurements including tissue Doppler imaging (TDI) and calculated indices in 26 male New Zealand white rabbits sedated with a combination of ketamine and midazolam

\begin{tabular}{lccc}
\hline Parameter & Mean $\pm \mathrm{SD}$ & 5th percentile & 95th percentile \\
\hline Doppler HR $(\mathrm{bpm})$ & $262.77 \pm 37.17$ & 213.01 & 329.03 \\
Ao max $(\mathrm{m} / \mathrm{s})$ & $0.86 \pm 0.12$ & 0.67 & 1.08 \\
PA max $\left._{\text {m }} / \mathrm{s}\right)$ & $0.78 \pm 0.12$ & 0.61 & 0.98 \\
Mitral $E(\mathrm{~m} / \mathrm{s})$ & $0.78 \pm 0.15$ & 0.60 & 1.05 \\
Mitral $A(\mathrm{~m} / \mathrm{s})$ & $0.55 \pm 0.11$ & 0.42 & 0.76 \\
Mitral $E: A$ & $1.44 \pm 0.16$ & 1.26 & 1.65 \\
IVRT $(\mathrm{ms})$ & $31.42 \pm 6.19$ & 23.77 & 39.94 \\
IVCT $(\mathrm{ms})$ & $25.00 \pm 3.68$ & 19.02 & 30.43 \\
LVET $(\mathrm{ms})$ & $95.72 \pm 10.21$ & 79.89 & 112.22 \\
Tei index & $0.60 \pm 0.10$ & 0.48 & 0.76 \\
$S_{\mathrm{m}}$ LW $(\mathrm{m} / \mathrm{s})$ & $0.11 \pm 0.02$ & 0.08 & 0.14 \\
$E_{\mathrm{m}}$ LW $(\mathrm{m} / \mathrm{s})$ & $0.16 \pm 0.05$ & 0.09 & 0.25 \\
$A_{\mathrm{m}}$ LW $(\mathrm{m} / \mathrm{s})$ & $0.09 \pm 0.03$ & 0.06 & 0.13 \\
$E_{\mathrm{m}}: A_{\mathrm{m}}$ LW & $1.83 \pm 0.43$ & 1.34 & 2.61 \\
$E: E_{\mathrm{m}}$ LW & $5.24 \pm 1.55$ & 3.43 & 8.03 \\
$S_{\mathrm{m}}$ septal $(\mathrm{m} / \mathrm{s})$ & $0.10 \pm 0.02$ & 0.07 & 0.12 \\
$E_{\mathrm{m}}$ septal $(\mathrm{m} / \mathrm{s})$ & $0.11 \pm 0.04$ & 0.07 & 0.19 \\
$A_{\mathrm{m}}$ septal $(\mathrm{m} / \mathrm{s})$ & $0.08 \pm 0.02$ & 0.04 & 0.12 \\
$E_{\mathrm{m}}: A_{\mathrm{m}}$ septal & $1.55 \pm 0.44$ & 0.65 & 2.22 \\
$E: E_{\mathrm{m}}$ septal & $7.75 \pm 2.69$ & 4.17 & 12.24 \\
\hline SD &
\end{tabular}

$\mathrm{SD}$, standard deviation; HR, heart rate; $\mathrm{Ao}_{\max }$, maximal aortic outflow velocity; $\mathrm{PA}_{\max }$, maximal pulmonary outflow velocity; Mitral $E$, maximal mitral $E$-wave velocity; Mitral $A$, maximal mitral $A$-wave velocity; IVRT, isovolumetric relaxation time; IVCT, isovolumetric contraction time; LVET, left ventricle ejection time; $S_{\mathrm{m}} \mathrm{LW}$, peak systolic mitral annular velocity from left wall; $E_{\mathrm{m}} \mathrm{LW}$, peak early diastolic mitral annular velocity from left wall; $A_{\mathrm{m}} \mathrm{LW}$, late early diastolic mitral annular velocity from left wall; $S_{\mathrm{m}}$ septal, peak systolic velocity of septal mitral annulus; $E_{\mathrm{m}}$ septal, peak early diastolic velocity of septal mitral annulus; and $A_{\mathrm{m}}$ septal, late early diastolic velocity of septal mitral annulus.

rate and $E_{\mathrm{m}} \mathrm{LW}(r=0.42, P=0.03)$ and $E_{\mathrm{m}}: A_{\mathrm{m}} \mathrm{LW}(r=$ $0.50, P=0.01)$. The bodyweight correlated weakly positive with Ao $(r=0.52, P<0.01)$ and weakly negative with $E_{\mathrm{m}}: A_{\mathrm{m}} \operatorname{septal}(r=0.44, P=0.03)$.

\section{Discussion}

Rabbits are an important model for cardiovascular research, mainly as they are small and relatively inexpensive but large enough to allow physiological experiments (Muders and Elsner, 2000). There are also various similarities between human and rabbit myocardium including a predomination of the $\beta$-myosin heavy-chain isoform, a positive force-frequency relationship and excitation-contraction coupling processes (Kavinsky et al., 1984; Ezzaher et al., 1992; Hasenfuss, 1998).

A complete Doppler echocardiographic examination including TDI evaluation was undertaken in rabbits anaesthetised with ketamine and midazolam. This anaesthetic combination yielded good immobilisation and allowed the ultrasonographer to obtain adequate 2D, M-mode, flow Doppler and TDI images for quantitative measurements. The combination is relatively common in research as well as in veterinary practice. In one study using this anaesthesia, some physiological and blood parameters were reduced (mean arterial pressure, $\mathrm{CO}_{2}$ arterial pressure) or not affected $\left(\mathrm{O}_{2}\right.$ arterial pressure $)$ relative to ketaminemidazolam-xylazine and tiletamine-zolazepam-xylazine anaesthesia (Dupras et al., 2001).

The mean heart rate observed in the present study was slightly higher than the range of mean heart rates previously reported for conscious rabbits (180-250 bpm) (Marano et al., 1996; Gil et al., 2004), and considerably higher when compared with rabbits anaesthetised with ketamine$\alpha_{2}$ agonists (medetomidine or xylazine) (Fontes-Sousa et al., 2006; Stypmann et al., 2007). Our results are in accordance with a previous study using rabbits, which found that ketamine-midazolam produced the highest heart rate compared with other anaesthetic combinations (Dupras et al., 2001). In fact, although these agents cause minimal cardiorespiratory depression, it has also been reported that they may lead to increased heart rate in humans (Marlow et al., 1991).

Some echocardiographic parameters are particularly sensitive to high heart rates, e.g., mitral $E$ - and $A$-wave that fuse and may not be distinguishable. Nevertheless, the use of high-speed tracing (100-200 m/s) allowed the assessment of $E$ - and $A$-wave peak flow velocities, even at elevated heart rates, but deceleration time of the $E$-wave could not be measured.

In recent years, TDI has emerged as a new modality that is less affected by loading conditions and so provides a strong complementary role in the assessment of diastolic function (Leite-Moreira, 2006). In the present study, mitral annulus velocity obtained from the septal and lateral (free wall) side with pulsed TDI was markedly higher than the values observed in awake rabbits or those anaesthetised with ketamine-xylazine (Stypmann et al., 2007). This higher mitral annulus velocity could be explained by sympathetic stimulation induced by ketamine (positive chronotropic and inotropic effects) and the minimal cardiovascular effects associated with midazolam anaesthesia (Dupras et al., 2001).

The ratio obtained between transmitral $E$ velocity and annular $E_{\mathrm{m}}, E: E_{\mathrm{m}}$ ratio, has been reported to be an accurate index of the level of filling pressure of the assessed ventricular chamber. In previous studies in humans this ratio had a strong correlation with pulmonary capillary wedge pressure (PCWP) and LV diastolic pressure (LVDP) (Nagueh et al., 1999; Ommen et al., 2000). In small animal medicine, it has been reported that an $E: E_{\mathrm{m}}$ value $>9.1$ indicated a mean left atrial pressure $>20 \mathrm{mmHg}$ in dogs with experimentally induced acute mitral regurgitation (Oyama et al., 2004).

Some potential limitations of the current study deserve attention, since we only used healthy anaesthetised rabbits. Firstly, the work only partially allowed us to assess the influence of the specific sedation used on the various echocardiographic parameters, since the same rabbits had not been examined in the conscious state. Nevertheless, some of the systolic and diastolic parameters were similar to those reported previously in the conscious state (fractional 
shortening, Tei index, and maximal mitral $E$ - and $A$-wave velocities) (Stypmann et al., 2007). Secondly, the work did not allow an assessment of the efficacy of the newer echocardiographic parameters, such as Tei index and TDI, or possible superiority above conventional parameters for detecting myocardial disease. This will have to be demonstrated in future studies in rabbits with induced or spontaneous cardiomyopathies.

Finally, we did not evaluate intra-operator variability. Poor repeatability has been reported in the acquisition of the velocities from the long-axis posterior wall and interventricular septum using pulsed TDI analysis (Simpson et al., 2007). Another study showed that the intra-examination variability was better under anaesthetised conditions (Chetboul et al., 2004), which was attributed to perfect immobility of the animal that improved repeatability of TDI measurements.

\section{Conclusions}

Echocardiographic reference values for New Zealand white rabbits anaesthetised with ketamine-midazolam are presented providing reference values for future studies. Emphasis was given to more recent indices that simultaneously reflect systolic and diastolic cardiac function, such as the Tei index derived from pulsed Doppler echocardiography and the pulsed TDI of the mitral annulus. Most of the results were comparable to those found in non-anaesthetised rabbits, and thus ketamine-midazolam anaesthesia may offer a good alternative when sedation is necessary.

\section{Conflict of interest statement}

There are no financial or other relations that could lead to a conflict of interest.

\section{Acknowledgments}

This work was supported by the Portuguese Foundation for Science and Technology (No. POCI/SAU-FCT/60803/ 2004) through Cardiovascular R\&D Unit (FCT nr. 51/94).

Ana Patrícia Fontes-Sousa is supported by a Grant from the Portuguese Foundation for Science and Technology (No. SFRH/BD/22590/2005). The authors would like to thank Pedro Leitão for technical support during echocardiographic examination.

\section{References}

Barraud, D., Faivre, V., Damy, T., Welschbillig, S., Gayat, E., Heymes, C., Payen, D., Shah, A.M., Mebazaa, A., 2007. Levosimendan restores both systolic and diastolic cardiac performance in lipopolysaccharidetreated rabbits: comparison with dobutamine and milrinone. Critical Care Medicine 35, 1376-1382.

Bras-Silva, C., Fontes-Sousa, A.P., Moura, C., Areias, J.C., LeiteMoreira, A.F., 2006. Impaired response to ET(B) receptor stimulation in heart failure: functional evidence of endocardial endothelial dysfunction? Experimental Biology and Medicine 231, 893-898.
Bruch, C., Schmermund, A., Dagres, N., Katz, M., Bartel, T., Erbel, R., 2002. Severe aortic valve stenosis with preserved and reduced systolic left ventricular function: diagnostic usefulness of the Tei index. Journal of the American Society of Echocardiography 15, 869-876.

Chetboul, V., Athanassiadis, N., Carlos, C., Nicolle, A., Zilberstein, L., Pouchelon, J.L., Lefebvre, H.P., Concordet, D., 2004. Assessment of repeatability, reproducibility, and effect of anesthesia on determination of radial and longitudinal left ventricular velocities via tissue Doppler imaging in dogs. American Journal of Veterinary Research 65, 909915 .

Chetboul, V., Sampedrano, C.C., Concordet, D., Tissier, R., Lamour, T., Ginesta, J., Gouni, V., Nicolle, A.P., Pouchelon, J.L., Lefebvre, H.P., 2005. Use of quantitative two-dimensional color tissue Doppler imaging for assessment of left ventricular radial and longitudinal myocardial velocities in dogs. American Journal of Veterinary Research 66, 953-961.

Chetboul, V., Sampedrano, C.C., Tissier, R., Gouni, V., Saponaro, V., Nicolle, A.P., Pouchelon, J.L., 2006. Quantitative assessment of velocities of the annulus of the left atrioventricular valve and left ventricular free wall in healthy cats by use of two-dimensional color tissue Doppler imaging. American Journal of Veterinary Research 67, 250-258.

Dujardin, K.S., Tei, C., Yeo, T.C., Hodge, D.O., Rossi, A., Seward, J.B., 1998. Prognostic value of a Doppler index combining systolic and diastolic performance in idiopathic-dilated cardiomyopathy. The American Journal of Cardiology 82, 1071-1076.

Dupras, J., Vachon, P., Cuvelliez, S., Blais, D., 2001. Anesthesia of the New Zealand rabbit using the combination of tiletamine-zolazepam and ketamine-midazolam with or without xylazine. The Canadian Veterinary Journal 42, 455-460.

Dyer, K.L., Pauliks, L.B., Das, B., Shandas, R., Ivy, D., Shaffer, E.M., Valdes-Cruz, L.M., 2006. Use of myocardial performance index in pediatric patients with idiopathic pulmonary arterial hypertension. Journal of the American Society of Echocardiography $19,21-27$.

Ezzaher, A., el Houda Bouanani, N., Crozatier, B., 1992. Force-frequency relations and response to ryanodine in failing rabbit hearts. The American Journal of Physiology 263, H1710-H1715.

Firstenberg, M.S., Greenberg, N.L., Main, M.L., Drinko, J.K., Odabashian, J.A., Thomas, J.D., Garcia, M.J., 2001. Determinants of diastolic myocardial tissue Doppler velocities: influences of relaxation and preload. Journal of Applied Physiology 90, 299-307.

Fontes-Sousa, A.P., Bras-Silva, C., Moura, C., Areias, J.C., LeiteMoreira, A.F., 2006. M-mode and Doppler echocardiographic reference values for male New Zealand white rabbits. American Journal of Veterinary Research 67, 1725-1729.

Gaber, C., 1991. Doppler echocardiography. Problems in Veterinary Medicine 3, 479-499.

Gaibazzi, N., Petrucci, N., Ziacchi, V., 2005. Left ventricle myocardial performance index derived either by conventional method or mitral annulus tissue-Doppler: a comparison study in healthy subjects and subjects with heart failure. Journal of the American Society of Echocardiography 18, 1270-1276.

Gan, L.M., Wikstrom, J., Brandt-Eliasson, U., Wandt, B., 2004. Amplitude and velocity of mitral annulus motion in rabbits. Echocardiography 21, 313-317.

Gil, A.G., Silvan, G., Illera, M., Illera, J.C., 2004. The effects of anesthesia on the clinical chemistry of New Zealand white rabbits. Contemporary Topics in Laboratory Animal Science 43, 25-29.

Haque, A., Otsuji, Y., Yoshifuku, S., Kumanohoso, T., Zhang, H., Kisanuki, A., Minagoe, S., Sakata, R., Tei, C., 2002. Effects of valve dysfunction on Doppler Tei index. Journal of the American Society of Echocardiography 15, 877-883.

Harjai, K.J., Scott, L., Vivekananthan, K., Nunez, E., Edupuganti, R., 2002. The Tei index: a new prognostic index for patients with symptomatic heart failure. Journal of the American Society of Echocardiography 15, 864-868. 
Hasenfuss, G., 1998. Animal models of human cardiovascular disease, heart failure and hypertrophy. Cardiovascular Research 39, 60-76.

Kavinsky, C.J., Umeda, P.K., Levin, J.E., Sinha, A.M., Nigro, J.M., Jakovcic, S., Rabinowitz, M., 1984. Analysis of cloned mRNA sequences encoding subfragment 2 and part of subfragment 1 of alpha- and beta-myosin heavy chains of rabbit heart. The Journal of Biological Chemistry 259, 2775-2781.

Lange, M., Smul, T.M., Blomeyer, C.A., Redel, A., Klotz, K.N., Roewer, N., Kehl, F., 2006. Role of the beta1-adrenergic pathway in anesthetic and ischemic preconditioning against myocardial infarction in the rabbit heart in vivo. Anesthesiology 105, 503-510.

Leite-Moreira, A.F., 2006. Current perspectives in diastolic dysfunction and diastolic heart failure. Heart 92, 712-718.

Marano, G., Grigioni, M., Tiburzi, F., Vergari, A., Zanghi, F., 1996. Effects of isoflurane on cardiovascular system and sympathovagal balance in New Zealand white rabbits. Journal of Cardiovascular Pharmacology 28, 513-518.

Marlow, R., Reich, D.L., Neustein, S., Silvay, G., 1991. Haemodynamic response to induction of anaesthesia with ketamine/midazolam. Canadian Journal of Anaesthesia 38, 844-848.

Martin, M.W., Darke, P.G., Else, R.W., 1987. Congestive heart failure with atrial fibrillation in a rabbit. Veterinary Record 121, 570-571.

Muders, F., Elsner, D., 2000. Animal models of chronic heart failure. Pharmacology Research 41, 605-612.

Nagueh, S.F., Lakkis, N.M., Middleton, K.J., Spencer 3rd, W.H., Zoghbi, W.A., Quinones, M.A., 1999. Doppler estimation of left ventricular filling pressures in patients with hypertrophic cardiomyopathy. Circulation 99, 254-261.

Nagueh, S.F., Sun, H., Kopelen, H.A., Middleton, K.J., Khoury, D.S., 2001. Hemodynamic determinants of the mitral annulus diastolic velocities by tissue Doppler. Journal of the American College of Cardiology 37, 278-285.

Oki, T., Tabata, T., Mishiro, Y., Yamada, H., Abe, M., Onose, Y., Wakatsuki, T., Iuchi, A., Ito, S., 1999. Pulsed tissue Doppler imaging of left ventricular systolic and diastolic wall motion velocities to evaluate differences between long and short axes in healthy subjects. Journal of the American Society of Cardiology 12, 308-313.

Ommen, S.R., Nishimura, R.A., Appleton, C.P., Miller, F.A., Oh, J.K., Redfield, M.M., Tajik, A.J., 2000. Clinical utility of Doppler echocardiography and tissue Doppler imaging in the estimation of left ventricular filling pressures: a comparative simultaneous Dopplercatheterization study. Circulation 102, 1788-1794.

O’Sullivan, M.L., O’Grady, M.R., Minors, S.L., 2007. Assessment of diastolic function by Doppler echocardiography in normal Doberman Pinschers and Doberman Pinschers with dilated cardiomyopathy. Journal of Veterinary Internal Medicine 21, 81-91.
Oyama, M.A., Sisson, D.D., Bulmer, B.J., Constable, P.D., 2004. Echocardiographic estimation of mean left atrial pressure in a canine model of acute mitral valve insufficiency. Journal of Veterinary Internal Medicine 18, 667-672.

Sahn, D.J., DeMaria, A., Kisslo, J., Weyman, A., 1978. Recommendations regarding quantitation in M-mode echocardiography: results of a survey of echocardiographic measurements. Circulation 58, 10721083.

Sanford, T.D., Colby, E.D., 1980. Effect of xylazine and ketamine on blood pressure heart rate and respiratory rate in rabbits. Laboratory Animal Science 30, 519-523.

Schaefer, A., Meyer, G.P., Brand, B., Hilfiker-Kleiner, D., Drexler, H., Klein, G., 2005. Effects of anesthesia on diastolic function in mice assessed by echocardiography. Echocardiography 22, 665-670.

Simpson, K.E., Devine, B.C., Gunn-Moore, D.A., French, A.T., DukesMcEwan, J., Koffas, H., Moran, C.M., Corcoran, B.M., 2007. Assessment of the repeatability of feline echocardiography using conventional echocardiography and spectral pulse-wave Doppler tissue imaging techniques. Veterinary Radiology and Ultrasound 48, $58-68$.

Sohn, D.W., Chai, I.H., Lee, D.J., Kim, H.C., Kim, H.S., Oh, B.H., Lee, M.M., Park, Y.B., Choi, Y.S., Seo, J.D., Lee, Y.W., 1997. Assessment of mitral annulus velocity by Doppler tissue imaging in the evaluation of left ventricular diastolic function. Journal of the American College of Cardiology 30, 474-480.

Stypmann, J., Engelen, M.A., Breithardt, A.K., Milberg, P., Rothenburger, M., Breithardt, O.A., Breithardt, G., Eckardt, L., Cordula, P.N., 2007. Doppler echocardiography and tissue Doppler imaging in the healthy rabbit: differences of cardiac function during awake and anaesthetised examination. International Journal of Cardiology 115, 164-170.

Tei, C., Ling, L.H., Hodge, D.O., Bailey, K.R., Oh, J.K., Rodeheffer, R.J., Tajik, A.J., Seward, J.B., 1995. New index of combined systolic and diastolic myocardial performance: a simple and reproducible measure of cardiac function - a study in normals and dilated cardiomyopathy. Journal of Cardiology 26, 357-366.

Teshima, K., Asano, K., Sasaki, Y., Kato, Y., Kutara, K., Edamura, K., Hasegawa, A., Tanaka, S., 2005. Assessment of left ventricular function using pulsed tissue Doppler imaging in healthy dogs and dogs with spontaneous mitral regurgitation. The Journal of Veterinary Medical Science 67, 1207-1215.

Thomas, W.P., Gaber, C.E., Jacobs, G.J., Kaplan, P.M., Lombard, C.W., Moise, N.S., Moses, B.L., 1993. Recommendations for standards in transthoracic two-dimensional echocardiography in the dog and cat. Echocardiography Committee of the Specialty of Cardiology, American College of Veterinary Internal Medicine. Journal of Veterinary Internal Medicine 7, 247-252. 